\title{
Docking of Dengue NS2B-NS3 Protease with Murraya koenigii
}

\author{
Kai Sing Yong ${ }^{1 *}$, Sy Bing Choi ${ }^{1,2}$, Habibah A. Wahab ${ }^{3}$ \\ ${ }^{I}$ School of Pharmaceutical Sciences, Universiti Sains Malaysia, 11800 USM, Pulau Pinang, Malaysia. \\ ${ }^{2}$ School of Industrial Technology, Universiti Sains Malaysia, 11800 USM, Pulau Pinang, Malaysia. \\ ${ }^{3}$ Malaysian Institute of Pharmaceuticals and Nutraceuticals, Kementerian Sains, Teknologi dan Inovasi Blok 5-A, Halaman Bukit Gambir, \\ 11700, Pulau Pinang, Malaysia.
}

Received: 3 September 2014 / Accepted: 30 November 2014

\begin{abstract}
:
Dengue disease has been reported for more than 100 million cases every year distressing tropical and subtropical countries. Dengue virus carries a positive single strand RNA, belongs to Flaviviridae family consist of four serotypes give rise to undifferentiated fever, dengue fever (DF), dengue hemorrhagic fever (DHF) or dengue shock syndrome (DSS). Currently, there is no licensed drug available for all serotypes and till date several vaccine candidates are still under development. In this study, in silico docking and virtual screening on the major dengue drug target namely serine protease was done against NADI database to elucidate the potential drug lead. Virtual screening results reflected that compounds from Murraya koenigii had significant inhibition potential towards serine protease. Thus, details investigation on the interaction mechanism was carried out using Autodock 4.2. The docking result indicated that the potential compound from M. koenigii show a favourable free energy of binding $(-7.81 \mathrm{kcal} / \mathrm{mol})$ towards the drug target. It was found that this compound is attributed to some essential interaction between residues such as Met84, Asp129, Phe130, Tyr150, Asn152, Gly153, Val154, Val155, and Tyr161. This finding has provided useful understanding for M. koenigii in developing potential inhibitor candidates for future studies in dengue.
\end{abstract}

Key words: Dengue, docking, NS2B-NS3 protease, Murraya koenigii

\section{Introduction}

Dengue infection is transmitted by Aedes mosquitoes and it affects tropical and subtropical countries. There are four serotypes of dengue known as DENV1, DENV2, DENV3 and DENV4 which genetically similar but different in serological field. Dengue disease can vary from mild and febrile dengue fever, to hemorrhagic fever and fatal shock syndrome [1,2]. The World Health Organization (WHO) estimated that of 500,000 severe dengue cases occurred annually and total of $2.5 \%$ lead to deaths.

In 2013, cases have occurred in Florida of US, Yunnan of China, Honduras, Costa Rica and Mexico of South American, Laos and Singapore of Asia. In Malaysia, it was reported a total of 43,346 dengue infections with $0.21 \%$ of death rates in 2013 alone [3]. The trends has become severe in 2014 as dengue virus type 3 (DEN3) affect the large population of human beings in Cook Islands, Malaysia, Fiji and Vanuatu after over a 10 years interval.

Dengue is caused by RNA virus named Flavivirus from family Flaviviridae. The virion genome encoded with three structural proteins (Capsid, Membrane, Envelope) and seven non-structural proteins (NS1, NS2A, NS2B, NS3, NS4A, NS4B, NS5). NS2B-NS3 protease is one of the important therapeutic targets because it is able to catalyze the viral processing polyprotein $[4,5]$.
NS3 hold two functional domains, an N-terminal serine protease with the size of 170 amino acids, and a Cterminal helicase or RNA triphosphatase with 440 amino acids combine with NS2B cofactor which has only 40 amino acids. Catalytic triad was found (His51, Asp75, and Ser135) conserved within the four serotypes of dengue acted as the active site.

Till date, there is yet to have approved drug candidate in the market. The vaccine candidate available at the moment consist of four recombinants, live, attenuated vaccine is developed under phase III clinical trial as of May 2014 [6,7]. In previous study, synthetic peptide-like inhibitors, substrate-like aldehydes [8,9] show good inhibition towards dengue virus. Cyclohexenyl chalcone derivatives from Bosenbergia rotunda show strong inhibition with value of inhibition constant, $\mathrm{K}_{\mathrm{i}}$ of $25 \mu \mathrm{M}$ [10]. Natural product has been proven that they can be the precursor for novel and drug lead candidate in the process of drug discovery for in vitro studies [11].

In this work, natural product from Murraya koenigii was investigated toward dengue inhibition. In silico approach was used here to study the interaction of dengue's serine protease against the compounds from Murraya koenigii.

\footnotetext{
*Corresponding author: Yong Kai Sing,

E-mail: kaisingyong@gmail.com
} 


\section{Experimental}

Structure of serine protease NS2B-NS3 was taken from Protein Data Bank (PDB ID: 3U1I) where N-benzoyl-Lnorleucyl-6- ammonio-L-norleucyl-N-5 [amino(iminio)methyl]-N-[(2S)-5-carbamimidam-ido-1-hydroxypentan2-yl]-L-ornithin-amide (Bz-Nle-Lys-Arg-Arg-H) is the peptide inhibitor in protein crystal structure. Covalent control docking is performed by incorporating the flexibility at the side chain of important interacting residues namely; SER135 at chain B was done. Docking procedure was carried out using Autodock Tools version 1.5.6 and AutoDock 4.2. The gasteiger charges were employed for both protein and ligand molecules. All non-polar hydrogens for both protein and ligand were merged. The map of $62 \times 62 \times 62 \AA$ grid points at $0.375 \AA$ spacing were generated using the AutoGrid 4 and centered on the potential binding site with the Cartesian coordination of 25.286, -16.66, 9.079. Molecular docking simulation was carried out using the Lamarckian genetic algorithm with AutoDock 4.2. The lowest free energy of binding of each conformation in the most populated cluster was selected. Interaction analyses were done and visualized using Discovery studio 2.5, Accelrys Software Inc.

\section{Results and Discussion}

The control docking was analyzed prior to the docking with the compounds from $M$. koenigii. Top ranked cluster with the lowest free energy of binding (FEB) and the root mean square deviation (RMSD) value of less than $2.0 \AA$ was validated. The FEB of the control dock was $-6.64 \mathrm{kcal} / \mathrm{mol}$ with the RMSD value of $1.3 \AA$ as shown in Table 1. In control docking, amino acids involved in pi-cation interactions are His51 and Tyr 161. Besides, residues Met84, Asp129, Gly133, Thr134, Ser135, Gly151, Asn152 and Gly153 take part in hydrogen bond formation. The Van der Waals interactions were observed in residues Gly82, Thr83, Arg85 from chain A and Asp75, Phe130, Tyr150, Val155 from chain B.

Six compounds from $M$. koenigii were docked using same parameter optimized from control docking and the results were summarized in Table 1 . It was found that the compound MSC68 and MSC88 can only occupy one of the binding pockets and the binding affinity is not favourable with -4.37 and $-6.03 \mathrm{kcal} / \mathrm{mol}$, respectively. Both terpenes do not have significant hydrogen bond interactions and pi-pi interactions with protease. MSC1300 has pi-cation interaction with Arg54 and hydrogen bond interaction with residue Thr83 which do not exhibit any interaction with serine protease as compared to the control docking studies. MSC1425 only form hydrogen bonds with residues Gly151 and Gly153 from S1, S2 and S4 pockets. This study focuses on the compounds having similar binding interaction in binding pockets as shown in Figure 1 with control docking study.
From the control docking results, residue Gly153 played an important role as hydrogen bond donor and acceptor with tetrapeptide inhibitor. In Figure 2, the similar binding mode is found when MSC1308 forms hydrogen bond with the oxygen atom from Gly153 as acceptor with bond length $2.2 \AA$. In addition, hydrogen attached to nitrogen atom of Gly153 acted as hydrogen bond donor that forms hydrogen bonding interaction with oxygen atom in MSC1308. Besides, oxygen atom of Sitoster-4-en-3-one (MSC1320) forms hydrogen bond with hydrogen atom of Trp50 at $2.0 \AA$.

Table 1. FEB of control docked complex and ligands

\begin{tabular}{|c|c|c|}
\hline Ligands & $\begin{array}{l}\text { Binding energy } \\
(\mathrm{kcal} / \mathrm{mol})\end{array}$ & Cover pocket \\
\hline $\begin{array}{l}\text { Control docked } \\
\text { inhibitor }\end{array}$ & -6.64 & S1, S2, S3, S4 \\
\hline MSC68 & -4.37 & S1 \\
\hline MSC88 & -6.03 & S1 \\
\hline MSC1300 & -7.64 & $\mathrm{~S} 2, \mathrm{~S} 3, \mathrm{~S} 4$ \\
\hline MSC1308 & -7.81 & $\mathrm{~S} 1, \mathrm{~S} 3, \mathrm{~S} 4$ \\
\hline MSC1320 & -8.20 & $\mathrm{~S} 1, \mathrm{~S} 2$ \\
\hline MSC1425 & -7.11 & $\mathrm{~S} 1, \mathrm{~S} 2, \mathrm{~S} 4$ \\
\hline
\end{tabular}

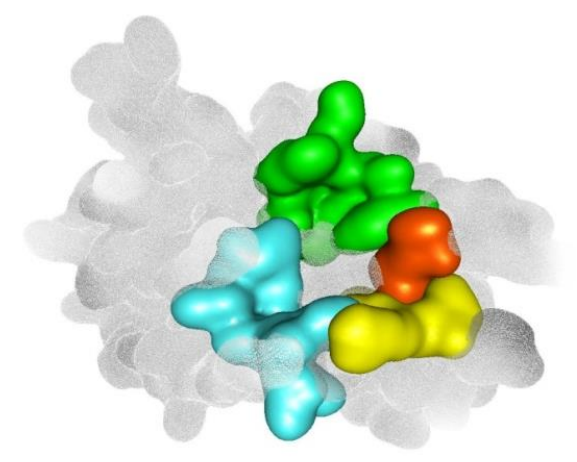

Figure 1. Binding pockets in serine protease: S1 (green), S2 (cyan), S3 (yellow) and S4 (orange).

Aromatic properties of amino acid tyrosine tend to form pi interactions with ligands. Therefore, carbazole rings of MSC 1308 were found with the formations of three pipi interactions with Tyr 161 with distance of $3.7 \AA, 3.8 \AA$ and $4.7 \AA$ respectively. In Figure 3, pi-sigma interaction was also formed between methyl group from the side chain of MSC1320 and Tyr161 at distance $3.9 \AA$. Besides, residue Val154 forms pi-sigma interaction with nitrogen atom in another five membered cylic ring of MSC 1308 with distance of $3.8 \AA$.

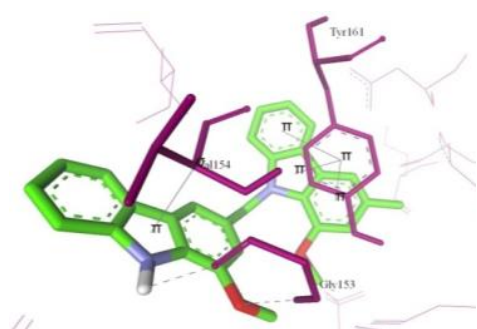

Figure 2. Binding interactions between MSC1308 and residues: pi-sigma interaction (Val154), three pi-pi interactions (Tyr161) and two hydrogen bonds (Gly153). 


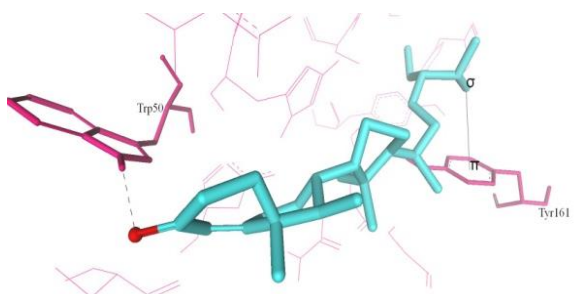

Figure 3. MSC1320 was found with a single hydrogen bond formed between ligand and Trp50 and pi-sigma interaction with Tyr161.

Bismurrayafoline E (MSC1308) has three pi-pi and one pi-sigma interactions and two hydrogen bond interactions with serine protease. The Van der Waals interactions formed around MSC1308 involving residues Met84, Asp129, Phe130, Tyr150, Asn152, and Val155. MSC1308 cover S1, S3 and S4 binding pockets while MSC1320 cover S1 and S2 binding pockets. The residues contribute to van der Waals interactions in MSC1320 are His51, Arg54, Val72, Asp75, Asp81, Pro132, Thr134, Ser135, Tyr150, Gly151 and Asn152 at scaling factor of $1.0 \AA$. Among all six compounds, MSC1308 had adopted similar binding interactions as compared to the inhibitor from control docking. Thus, MSC1308 can be postulated as a potential inhibitor among the other five compounds stated above.

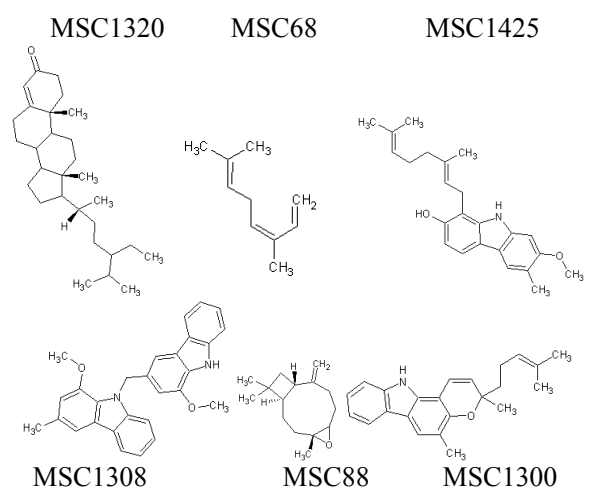

Figure 4. Structure of ligands in Murraya koenigii.

\section{Conclusions}

From this study, docking results revealed that $\mathrm{S} 1$ and $\mathrm{S} 2$ pocket on DEN3 NS2B-NS3 protease are very important for ligand binding specificity. It was also found that in order to have a good ligand binding affinity, hydrogen bond, pi interactions and van der Waals interactions had make an important contribution for the binding interactions between compound and protease. This was shown by MSC1308 from M. koenigii which has similar binding interactions with inhibitor control docking. Thus, as a concluding remark, MSC1308 could be a potent inhibitor for DEN3 NS2B-NS3 protease and this work. Further extend to experimental study by investigating on how ligands inhibit the protease via in vitro assay can be done in the future.

\section{Acknowledgement}

Support of ScienceFund Mosti with grant number 304/PFARMASI/650600/I121 and University Sains Malaysia for support in the form of postgraduate fellowship, are gratefully acknowledged.

\section{References}

[1] C.G. Noble, C. Yen-Liang, D. Hangping, and G. Feng, Strategies for development of dengue virus inhibitors, Antiviral Research, 85, 2010, 450-62.

[2] D.J. Gubler, Epidemic dengue / dengue hemorrhagic fever as a public health, social and economic problem in the 21 st century, Trends in Microbiology, 10(2), 2002, 100-103.

[3] http:// www.wpro.who.int/ emerging_diseases/ DengueSituationUpdates /en/

[4] N.H. Mueller, C. Yon, V.K. Ganesh, and R. Padmanabhan, Characterization of the West Nile virus protease substrate specificity and inhibitors, The International Journal of Biochemistry \& Cell Biology, 39, 2007, 606-614.

[5] G. Katzenmeier, Inhibition of NS2B-NS3 protease - towards a causative therapy for Dengue virus disease, Dengue Bulletin, 28, 2004, 58-67.

[6] World Health Organization (2014), Fact sheet: Dengue and severe dengue. Accessible online at http://www.who.int/mediacentre/factsheets/fs117/en/index.ht $\mathrm{ml}$

[7] Special Issue: The Development of Dengue Vaccines, Vaccine, 29(42), 2011, 7219-7284.

[8] S. Chanprapaph, P. Saparpakorn, C. Sangma, P. Niyomrattanakit, S. Hannongbua, C. Angsuthanasombat, and G. Katzenmeier, Competitive inhibition of the dengue virus NS3 serine protease by synthetic peptides representing polyprotein cleavage sites, Biochemical and Biophysical Research Communications, 330(4), 2005, 1237-1246.

[9] D. Leung, K. Schroder, H. White, N. -X. Fang, M. J. Stoermer, G. Abbenante, J.L. Martin, P.R. Young, and D.P. Fairlie, Activity of recombinant dengue 2 virus ns 3 protease in the presence of a truncated ns $2 \mathrm{~b}$ co-factor, small peptide substrates, and inhibitors, Journal of Biological Chemistry, 276(49), 2001, 45762-45771.

[10] T.S. Kiat, R. Pippen, R. Yusof, H. Ibrahim, N. Khalid, and N. A. Rahman, Inhibitory activity of cyclohexenyl chalcone derivatives and flavonoids of fingerroot, Boesenbergia rotunda (L.), towards dengue-2 virus NS3 protease, Bioorganic \& Medicinal Chemistry Letters, 16(12), 2006, 3337-3340.

[11] M.S. Butler, The role of natural product chemistry in drug discovery, Journal of Natural Products, 67(12), 2004, 21412153. 